\title{
Effect of Overlapping Projections on Reconstruction Image Quality in Multipinhole SPECT
}

\author{
Kathleen Vunckx and Johan Nuyts
}

\begin{abstract}
Multipinhole imaging has a lot of advantages over single pinhole, such as an increased sensitivity and an improved sampling. However, the quest for a good design is tedious, due to the large number of design parameters. One of these, the amount of overlap in the projection images, and its effect on the reconstruction image quality, is examined in this paper. The evaluation of the quality is based on efficient approximations for the linearized local impulse response and the covariance in a voxel. Two (theoretical) methods for overlap removal and two measures to quantify the overlap are proposed. First, the approximate method is validated with standard iterative reconstructions. Second, designs with different amounts of overlap are evaluated before and after overlap removal to verify the effect of multiplexing. Third, the results are interpreted with the overlap quantification measures. We can conclude that overlap removal improves image quality: the loss of information due to multiplexing is not compensated by the increase in sensitivity.
\end{abstract}

Index Terms-Multipinhole SPECT, overlap, multiplexing, reconstruction image quality.

\section{INTRODUCTION}

Over the past decade research on small animal imaging has gained a lot of interest. For SPECT, single pinhole collimation is often used. However, because of its low sensitivity, multipinhole SPECT is receiving increased attention. However, the complexity of designing such a multipinhole collimator increases exponentially with the number of pinhole apertures. One of these design parameters is the amount of overlap (also called multiplexing) allowed for the projections through the different pinhole apertures. Two extreme cases are coded aperture collimation with a high degree of multiplexing (Meikle et al. [1]) and no overlap (Beekman et al. [2]), with in between a moderate amount of overlap (Schramm et al. [3]). Although this overlap has been studied previously (e.g. [4]-[6]), its effects are not yet completely understood. In this work we try to find the answer to the question: "Is it better to have more sensitivity thanks to overlapping projections or to remove the overlap (and thus reduce the sensitivity) in order to have unambiguous information in each detector pixel?" Therefore, we investigate the effect of multiplexing on the reconstruction image quality of each voxel. As a measure of image quality, the contrastto-noise ratio (CNR) of the linearized local impulse response (LLIR) in (some of) the voxels of the reconstructed image is used. For that purpose, we based ourselves on the method

Dept. of Nuclear Medicine, K.U.Leuven, B-3000 Leuven, Belgium.

This work is supported by F.W.O. grant G.0174.03, K.U.Leuven grant IDO/02/012, I.W.T. grant SBO - ANIMONE and 2006 IEEE NSS-MIC Trainee Award. presented in [7]. A description of this method can be found in section II-A. In section II-B we propose two (theoretical) ways to remove the overlap between the projections. To analyze the results, it is useful to have some measures that quantify the amount of overlap. Some suggestions are made in section IIC. In section III some simulation studies are described, the results of which are discussed in section IV. First a validation study is presented. Second, the effect of overlap on the image quality is studied based on a number of multipinhole designs with a varying degree of multiplexing. These designs are compared before and after overlap removal. Last, the overlap quantification measures are used to explain some of the results.

\section{THEORY}

\section{A. Reconstruction Image Quality Quantification}

To quantify image quality, the properties of the impulse response in each voxel are examined. However, since for emission tomography the reconstruction of the unknown activity distribution is space-variant, the standard impulse response theory cannot be applied. As a generalization of the impulse response for emission tomography the linearized local impulse response (LLIR) $l^{j}$ was proposed by Fessler et al. [8]:

$$
\begin{aligned}
l^{j}(\hat{\Lambda}) & =\lim _{\delta \rightarrow 0} \frac{\hat{\Lambda}\left(\bar{Q}\left(\Lambda+\delta e^{j}\right)\right)-\hat{\Lambda}(\bar{Q}(\Lambda))}{\delta} \\
& =\frac{\delta}{\delta \lambda_{j}} \hat{\Lambda}(\bar{Q}(\Lambda))
\end{aligned}
$$

where $\bar{Q}$ is the expectation of the projection data $Q, \hat{\Lambda}$ is the reconstruction of the unknown activity distribution $\Lambda, e^{j}$ is the $j$-th unit vector, and $j$ the number of the voxel in the reconstruction image.

The calculation of the LLIR and its covariance for each voxel $j$ using an iterative reconstruction method and multiple noise realisations, called reference method in the rest of this paper, is, however, very time-consuming. Therefore some efficient analytical approximations were proposed by Fessler et al. [8], [9] and Qi et al. [10] for converged maximum a posteriori (MAP) reconstruction:

$$
\begin{aligned}
l^{j}(\hat{\Lambda}) & \approx[\boldsymbol{F}+\beta \boldsymbol{U}]^{-1} \boldsymbol{F} e^{j} \\
\operatorname{Cov}^{j}(\hat{\Lambda}) & \approx[\boldsymbol{F}+\beta \boldsymbol{U}]^{-1} \boldsymbol{F}[\boldsymbol{F}+\beta \boldsymbol{U}]^{-1} e^{j}
\end{aligned}
$$

where $\boldsymbol{F}$ is the Fisher information matrix, which is a function of $\bar{Q}, \beta$ is the smoothing parameter and $\boldsymbol{U}$ is the Hessian of the quadratic prior, used for regularization. 
Although $F$ is shift variant, assuming local shift invariance only introduces a small error near the impulse in voxel $j$. The Fisher information matrix can thus be approximated by replacing its rows by shifted versions of the $j$-th row. This way $F$ is turned into a circulant matrix and it corresponds to a convolution, or a multiplication in the Fourier domain.

To be able to easily compare different pinhole collimator designs, we choose to impose a fixed target resolution in the reconstruction image. This is in fact equivalent to the analytical approach to get an isotropical spatial resolution presented in [11]. We replaced the MAP reconstruction by post-smoothed MLEM (maximum likelihood expectation maximization), with a Gaussian post-smooth filter $P$. This filter $P$ is chosen such that it makes the following approximation as accurate as possible: $T \approx P G F$, with $T$ a Gaussian with a full-width at half-maximum (FWHM) equal to the target resolution and $G$ the pseudoinverse of $F$. The LLIR and the covariance of voxel $j$ are then (as in [7]):

$$
\begin{aligned}
l^{j}(\hat{\Lambda}) & \approx \boldsymbol{P} \boldsymbol{G F} e^{j} \\
\operatorname{Cov}^{j}(\hat{\Lambda}) & \approx \boldsymbol{P} \boldsymbol{G F G P} \boldsymbol{P}^{\boldsymbol{T}} e^{j}
\end{aligned}
$$

where ${ }^{T}$ denotes transpose.

We have slightly adapted the convolution represented by $F$, by multiplying its elements with a weight that decreases with increasing distance from $j$, because the approximations are good close to $j$ and become less correct if the distance from $j$ increases.

The pseudoinverse of $F$ is calculated as follows:

$$
G=\frac{F^{*} \max (\Re(F), 0)}{F^{*} F \max (\Re(F), 0)+\epsilon}
$$

with $F^{*}$ the complex conjugate of $F, \Re(F)$ the real part of $F$ and $\epsilon=10^{-8}\left|F_{D C}\right|$, where $F_{D C}$ denotes the DC-value of $F$ in the Fourier domain, or the mean value of the corresponding image in the spatial domain.

As measures for the reconstruction quality in voxel $j$ the contrast recovery coefficient $(\mathrm{CRC})$ and the variance of the LLIR in voxel $j$ (which are the $j$-th element of $l^{j}$ and $\mathrm{Cov}^{j}$, respectively) are commonly used. Thanks to the fixed resolution after post-smoothing, the CRC is constant. The only parameter to optimize is then the variance. As an alternative the contrastto-noise ratio (CNR) could be optimized as well, since

$$
C N R=\frac{C R C}{\sqrt{\text { variance }}} .
$$

In the rest of this paper the CNR is taken as image quality measure.

\section{B. Overlap Removal Methods}

The amount of overlap in the projection images can be varied in different ways, such as:

- by varying the number of pinhole apertures,

- by varying the distance between the pinhole apertures,

- by varying the acceptance angle,

- by varying the aperture diameter, or a combination of the above. However, these methods make it harder to see the effect of an increased or decreased overlap on a voxel basis. Therefore, in addition, for each design two cases are compared. In the first case all overlap is tolerated, in the second one the overlap is entirely removed. In this section, two (theoretical) methods for overlap removal are presented.

The sensitivity of an aperture will be measured as the projection image of a uniform plane source through that particular aperture. Such a projection will be called a "sensitivity image", or simply the "sensitivity" of the corresponding aperture. (Note that this "sensitivity image" only provides the sensitivity as a function of two dimensions. It does not include the effect of the distance between the point source and the pinhole plane, and differs thus from the more commonly used definition of sensitivity).

The sensitivity is readily calculated using a ray-tracing technique similar to the method described by Schramm et al. [3], but originated from the detector pixel, instead of from the object voxel. Knowing the sensitivity for each aperture in each detector pixel, it is straightforward to detect and/or remove overlapping sensitivities in simulation experiments.

The two overlap removal methods, proposed here and used to compare designs with and without multiplexing, are:

1) Maximum sensitivity: The most obvious way to remove multiplexing is to keep the highest sensitivity in each detector pixel. All other sensitivities in that pixel are put to zero. This seems intuitively a good solution, since sensitivity and reconstruction image quality go hand in hand. This principle is illustrated in figure 1(a), where the colored curves represent profiles through the sensitivity images of the three pinhole apertures, and the white curve depicts a profile through the total sensitivity image after overlap removal with the maximum sensitivity method.

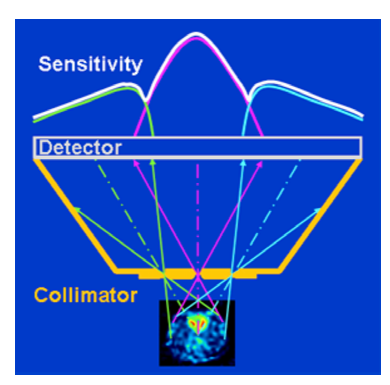

(a) Maximum sensitivity

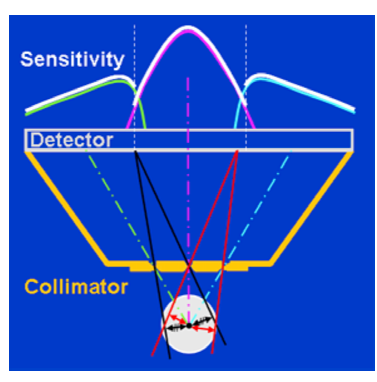

(b) Central sampling
Fig. 1. Illustration of the two proposed overlap removal methods.

2) Central sampling: Another useful approach could be to optimize the performance for the central field-of-view (FOV), by giving preference to extra sampling of central voxels over sampling of more excentrical voxels. For that purpose, the distance between the reconstruction image center and the backprojection lines starting from the detector pixel through the center of each pinhole aperture is computed. The sensitivity of the 
aperture corresponding to the smallest distance is preserved, the others are set to zero. For an illustration, see figure 1(b), where for one detector pixel in the overlap zone the backprojection lines through the two involved apertures are drawn in red. The backprojection line through the central pinhole lies closer to the image center than the line through the right pinhole. Therefore the sensitivity of the central aperture is kept, whereas that of the right aperture is put to zero. The black backprojection lines correspond to a detector pixel where the transistion of one sensitivity to another takes place, since the distances to the center of the image are equal.

\section{Overlap Quantification Measures}

The most common method to quantify the amount of multiplexing consists of calculating the ratio of the number of detector pixels that gather information from more than one pinhole to the total number of detector pixels gaining information from the object. This is a basic approach with several disadvantages. Firstly, it does not take into account the degree of multiplexing, i.e. how many pinhole apertures contribute to the overlap. Secondly, it only gives the global amount of overlap in the detector space. It is not related to the voxels, such that it is impossible to know which voxels and how many might suffer from this ambiguous information collection.

Therefore overlap quantification is envisaged from a different perspective in this paper. Since the main purpose of this work is to investigate the influence of overlapping projections on each reconstructed voxel, the measure that gives us an idea of the amount of overlap should also take every image voxel seperately into consideration.

The following measure of overlap is proposed:

$$
\phi_{j}=\frac{U_{j}}{U_{j}+M_{j}},
$$

where $j$ is the voxel under consideration, $U_{j}$ represents the "useful" sensitivity and $M_{j}$ stands for the "multiplexed" sensitivity. To compute $U_{j}$, a point source in $j$ is projected through each of the apertures, and the sensitivities at the corresponding detector pixels are accumulated. This gives an idea of the maximum amount of information that might be available during reconstruction. However, at these same detector pixels, activity, originating from other image voxels, can - in the case of multiplexing - also be seen through other apertures. Therefore, the sensitivities of the other apertures at these detector pixels are summed as well, yielding $M_{j}$.

As a result, $\phi_{j}$ is 1 in every voxel $j$ for non-multiplexing designs, and smaller than one if the sensitivities of different apertures overlap. In addition, a higher degree of multiplexing reduces $\phi_{j}$, as well in the case were overlap is created by an increasing number of apertures, as in the case were the share of the multiplexed sensitivity proportionally becomes bigger with respect to that of the useful sensitivity.

Another issue that should be taken into consideration, however, is the fact that overlap removal can reduce the total sensitivity for a voxel, because one or more projection lines are blocked by septa. Therefore we suggest a second measure ${ }^{1}$ :

$$
\psi_{j}=\frac{U_{j}^{\prime}}{U_{j}}
$$

with $U_{j}^{\prime}$ the total "useful" sensitivity for voxel $j$ after removing overlap. Consequently, $\psi_{j}$ equals one if the introduction of septa does not affect the sensitivity for voxel $j$, and decreases with the loss of "useful" sensitivity.

\section{Simulation SETUPS}

\section{A. Validation of the Image Quality Quantification}

In section II-A some efficient approximations for the LLIR in an image voxel and its covariance were described to quantify the image quality after post-smoothed MLEM reconstruction. Before using these approximations, it is appropriate to verify their accuracy with respect to the - very slow - reference method using post-smoothed MLEM reconstructions. This latter method requires 2 reconstructions of the noiseless object (one with and one without an impulse in the voxel of interest) to calculate the LLIR in one voxel. For each extra voxel an additional reconstruction is necessary to find its LLIR. The calculation of the covariance matrix costs $x$ more reconstructions to get a relative error on the standard deviation of $\sqrt{1 /(2 x)}$, irrespectively of the number of voxels under consideration.

As a validation study, 10 multipinhole designs with each 7 pinholes with an aperture diameter varying from $0.6 \mathrm{~mm}$ to $2.4 \mathrm{~mm}$ have been evaluated using both methods for comparison. The multiplexing case as well as the non-multiplexing case have been considered. The parameters ${ }^{2}$ of the multipinhole SPECT system are as follows: the distances from the detector to the center of the pinhole plate and to the axis-of-rotation (AOR) are $57 \mathrm{~mm}$ and $72 \mathrm{~mm}$, respectively. The detector size is $86 \times 70$ pixels, with a pixel size of $1.95 \mathrm{~mm}$ in both directions. The intrinsic resolution is taken $4.0 \mathrm{~mm}$. The 6 apertures surrounding the central one lie on a circle with a radius of $13 \mathrm{~mm}$. All apertures focus on the center-of-rotation (COR) and have an acceptance angle of $60^{\circ}$.

In a homogeneous sphere with a radius of $7 \mathrm{~mm}$ and an activity of $5 \mathrm{kBq} / \mathrm{mm}^{3}$ two voxels have been examined: one central point at $[0,0,0]$ and one excentrical point at $[0,2.4,4.0]$ (in $\mathrm{mm}$ ) from the image center. The simulated SPECT acquisition consisted of 64 projection angles (each $60 \mathrm{~s}$ ) measured over $360^{\circ}$. The cubic reconstruction voxels have a size of $0.8 \mathrm{~mm}$ and a target resolution of $2.4 \mathrm{~mm}$ is imposed.

For all simulations presented in this paper the resolution was modeled with the 7-ray resolution recovery method as decribed by Andreyev et al. [12]. The sensitivity was analytically calculated with a ray-tracing method, as mentioned in section II-B. However, for both septal penetration was neglected, since it was not yet included in the resolution recovery method. No

\footnotetext{
${ }^{1}$ For both measures the average has been taken over all projection angles.

${ }^{2}$ These parameters have been rescaled with respect to the parameters of a realistic multipinhole system on a clinical gamma camera to reduce the computation time.
} 
attenuation correction was done for these simulations, but it could be included very easily. Although we do not expect large differences, because of the small amount of attenuation caused by a rat, we plan to include it in the near future.

The iterative reconstructions were accelerated due to the use of the ordered subset algorithm [13]. An equivalent of 472 iterations were calculated to run MLEM close to convergence. The FWHM of the post-smooth filter was chosen to approach the target resolution as close as possible. For the calculation of the (co)variance 300 noise realisations were simulated.

\section{B. Effect of Overlap on Reconstruction Image Quality}

To investigate the effect of overlap on the reconstruction image quality, we compare several realistic multipinhole designs with different degrees of multiplexing. For each design, the CNR is calculated for a number of equally spaced voxels in a homogeneous sphere with a diameter of $35 \mathrm{~mm}$ (as shown in figure 2(a)), using the approximative method (see section II-A). Afterwards, the same designs were also tested for a software rat brain phantom with realistic dimensions and activities (see figures 2(b)-(d)) to verify that the same trends could be found for a different (and more relevant) phantom.

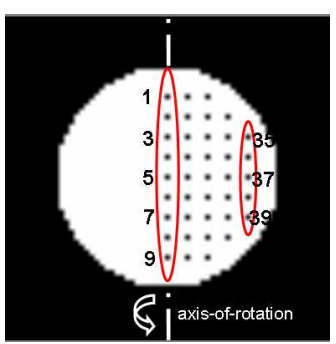

(a) Homogeneous sphere, transaxial

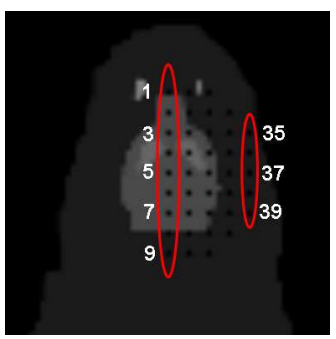

(c) Rat brain phantom, horizontal

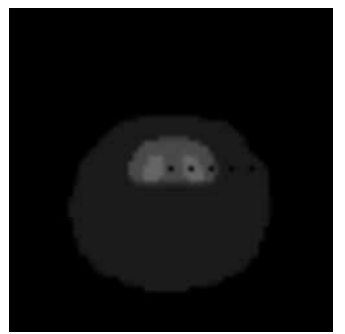

(b) Rat brain phantom, axial

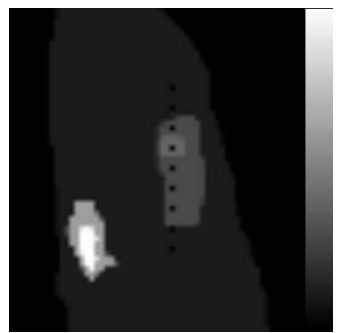

(d) Rat brain phantom, vertical
Fig. 2. The investigated voxels (a) in a homogeneous sphere with a diameter of $35 \mathrm{~mm}$ and (b)-(d) in a realistic software rat brain phantom. All voxels lie in a slice through the axis of rotation (AOR).

All investigated designs have the following main parameters. The distance between the detector and the pinhole plate is $173 \mathrm{~mm}$, whereas the distance from the detector to the AOR is $218 \mathrm{~mm}$. The detector consists of $256 \times 200$ square pixels, with a pixel size of $1.95 \mathrm{~mm}$ and an intrinsic resolution of $4.0 \mathrm{~mm}$. All pinhole apertures focus on the image center and are positioned on a circle with radius pinrad ${ }^{3}$ around a central pinhole. The aperture diameter is $1.2 \mathrm{~mm}$ and their acceptance angle is $60^{\circ}$. Again 64 projection views of $60 \mathrm{~s}$ each, measured over $360^{\circ}$ are simulated. The reconstruction voxel size is $0.8 \mathrm{~mm}$ and the target resolution is fixed to $2.4 \mathrm{~mm}$.

Three groups of multipinhole designs have been examined. The first group consisted of three designs with an increasing number of pinholes (5, 10 and 15 apertures), and thus an increasing amount of overlap. For each design the pinrad was $25 \mathrm{~mm}$. For the second group three 10-pinhole designs were investigated of which the distance between the apertures was varied (a pinrad of 15, 25 and $35 \mathrm{~mm}$ ). In this case the overlap decreases. The last group contained four designs, again with a varying number of apertures (9 to 15), but with a slightly different positioning with respect to the first two groups. The pinhole apertures surrounding the central one were alternatingly divided over two concentrical circles, the first with pinrad $20 \mathrm{~mm}$, the second with pinrad $30 \mathrm{~mm}$. This was done to improve the filling and the usage of the detector.

All designs have also been investigated for the case where the overlap was completely removed, using one of the two methods described in section II-B. In this way two kinds of overlap reduction are examined, i.e. a reduction by decreasing the number of pinholes or by putting the apertures farther apart, and a removal by e.g. placing septa to avoid overlap.

\section{Interpretation of the Effect of Overlap}

In this experiment the effect of (the amount of) overlap in the projection images, as well as the influence of overlap removal, will be investigated with the help of the two new measures for overlap quantification, described in section II-C. For this purpose, three 7-pinhole designs with a varying degree of multiplexing are examined using the approximative method (section II-A). Afterwards, these same three designs have been revised after removing the overlap.

The main system parameters are equal to the parameters stated above (section III-B). The pinrad ranges from $15 \mathrm{~mm}$ (large overlap) to $35 \mathrm{~mm}$ (almost no overlap). The CNR, $\phi_{j}$ and $\psi_{j}$ have been calculated for the same voxels of the homogeneous sphere described previously and depicted in figure 2.

\section{RESUlTS}

\section{A. Validation of the Image Quality Quantification}

The results of the validation study, presented in section III-A, are shown in figure 3 . For each design, the CNR in a central and an excentrical voxel and calculated with both methods is plotted with respect to the corresponding pinhole aperture diameter. The data obtained from the reference method are depicted in black, the ones calculated using the approximative method are

\footnotetext{
${ }^{3}$ With pinrad we mean the distance between the central pinhole aperture and the surrounding apertures, that all lie on the same concentrical circle.
} 
drawn in red. The diamonds denote the results for the central point, the stars indicate the outcome for the excentrical point.

When the new approximative method is compared to the reference method (post-smoothed MLEM), a good match can be seen as well for the quantitative values as for the optimal aperture diameter that can be derived from these curves. The optimum is found at 1.2-1.4 mm, which is much smaller than the imposed target resolution. This is in agreement with the findings of Fessler in [14].

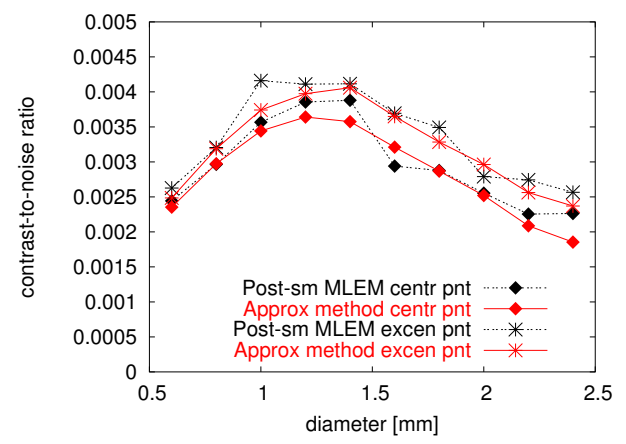

(a) With overlap

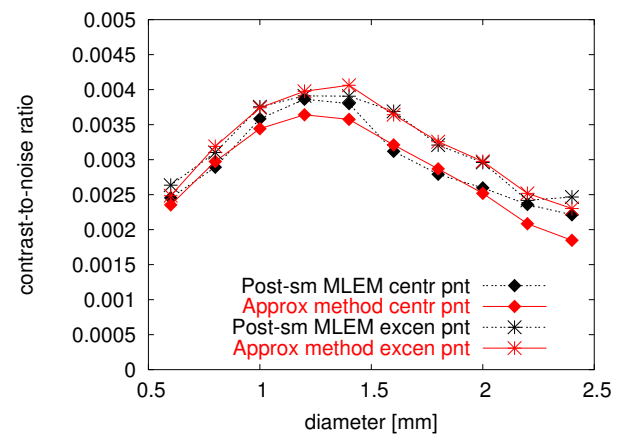

(b) No overlap

Fig. 3. Validation of the approximations for the quantification of the reconstruction image quality. The quality of several 7-pinhole designs with varying aperture diameter is evaluated, both in a central and an excentrical point in a homogeneous sphere. (a) With overlap, (b) overlap removed using the central sampling method.

In figure 3(a) designs are compared where overlapping projections are allowed. Figure 3(b) gives the results for the same designs, but for this experiment the overlap has been removed using the 'central sampling' technique (see section IIB.2). These graphs give almost identical results, which indicates that the overlap in the examined voxels (only present for the excentrical voxel) has little or no influence on their image quality.

\section{B. Effect of Overlap on Reconstruction Image Quality}

From the results of the study presented in section III-B we will try to find an answer to the question: is a large amount of overlap in the projections benificial or should it be reduced, and if so, how?

Figure 4(a) shows the CNR in the examined voxels of the homogeneous sphere of figure 2(a) for the first group of multipinhole designs. From this graph, a lot can be learned already about the effect of multiplexing. First of all, it can be seen that in the case where the overlap is maintained (solid lines), increasing the number of apertures is good up to a certain number. Adding more pinholes deteriorates the quality, probably due to the increased overlap and according incertainty about the origin of the activity detected in a detector pixel. A second point worth noting is the fact that this saturation in number of apertures does not occur (or at least at a much higher number) if the overlap is removed ${ }^{4}$ (dashed lines). However, this quality improvement is mostly the case for central voxels. The most (axially) excentrical voxels show little increase in reconstruction quality or even a small decrease. The reasons for these effects will be discussed in more detail in the next section. Last, but not least, it should be mentioned that all designs perform better after overlap removal. For the 5-pinhole design almost no difference can be seen, because already little multiplexing was present before overlap removal.

Similar conclusions, but from a different perspective, can be drawn from the second group of designs (see figure 4(b)). In this second part of the study the difference in amount of overlap is caused by a variation in the positioning of the pinhole apertures. In the first design the apertures lie much closer to the central one than in the last design, which corresponds to more multiplexing in the former case with respect to the latter case. From figure 4(b) again it can be noticed that for the overlapping designs the amount of overlap should be kept small. This conclusion is derived from the fact that a large distance between the pinholes (pinrad $35 \mathrm{~mm}$ ) gives the best image quality. For the non-multiplexed designs the pinholes can be put closer to each other, i.e. $15 \mathrm{~mm}$ pinrad if only the central voxels are of interest, but rather $25 \mathrm{~mm}$ pinrad if a more uniform quality is desired. Also from these results a general improvement can be seen if the overlap is removed, except for some (axially) very excentrical points. The largest difference occurs of course for the biggest overlap reduction.

The last group of designs aimed to improve the reconstruction image quality by a more sensible usage of the detector area. Due to the distribution of the pinhole apertures over two circles, instead of one, more (interesting) information is to be expected in the non-overlapping parts of the projection images. From figure 4(c) it can be seen that adding more apertures to the designs allowing overlap has almost no influence on the (uniform) image quality. If the overlap is removed, however, an increase in reconstruction quality more of less proportional to the additional number of pinholes can be perceived. This good result comes at the sacrifice of a decreased quality for some excentrical points.

In conclusion, we compare the results of the 15-pinhole

${ }^{4}$ For this study the central sampling overlap removal method, described in section II-B.2 is used for all designs. 


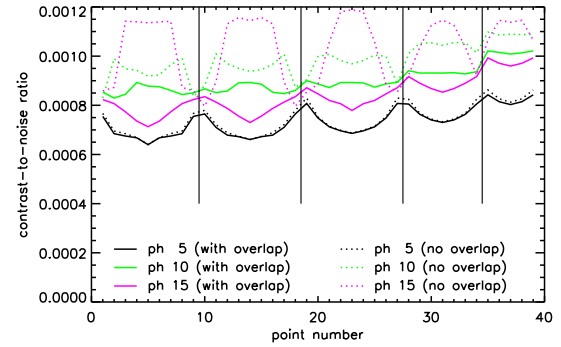

(a) Sphere, no. of pinh. 5-15, pinrad $25 \mathrm{~mm}$

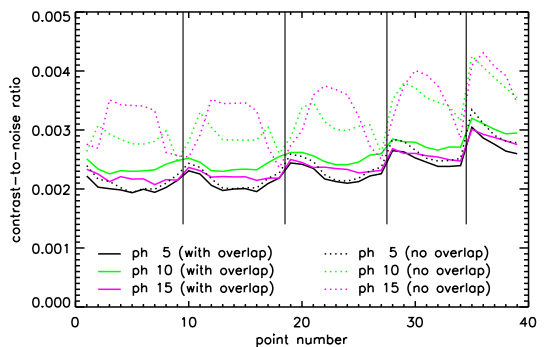

(d) Rat, no. of pinh. 5-15, pinrad $25 \mathrm{~mm}$

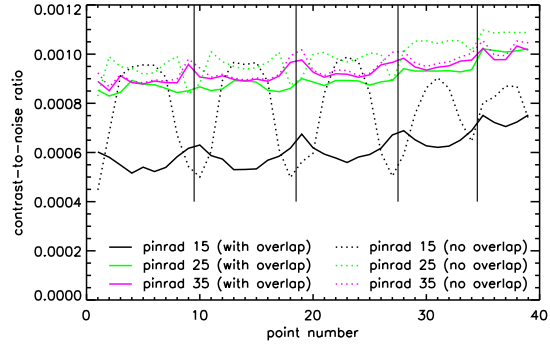

(b) Sphere, no. of pinh. 10, pinrad 15-35 mm

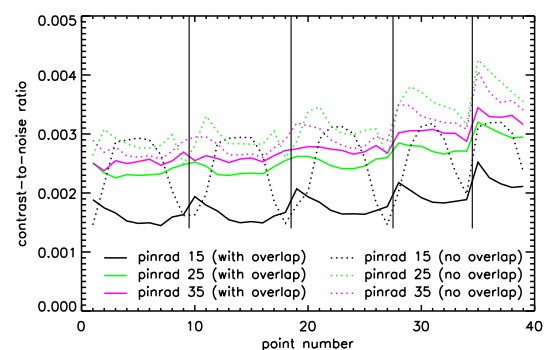

(e) Rat, no. of pinh. 10, pinrad 15-35 mm

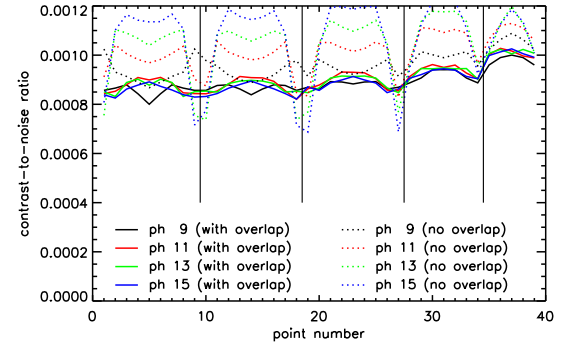

(c) Sphere, no. of pinh. 9-15, pinrad 20/30 mm (alt.)

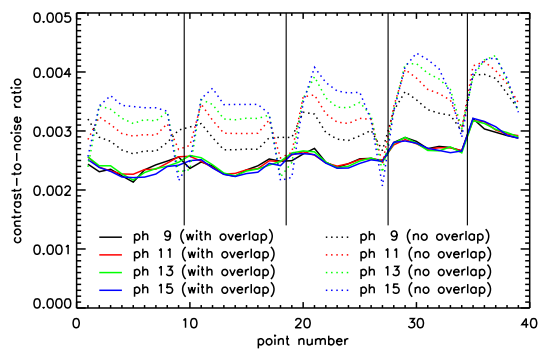

(f) Rat, no. of pinh. 9-15, pinrad 20/30 mm (alt.)

Fig. 4. Evaluation of three groups of multipinhole designs with different degrees of overlap and without overlap to examine the effect of overlap on the reconstruction image quality. The voxel indicated in figure 2 are plotted with respect to their CNR. The three upper graphs (a)-(c) plot the reconstruction quality of a homogeneous sphere, the three lower graphs (d)-(f) show the quality of a rat brain phantom. In (a) and (d) the number of apertures is varied from 5 to 15 and pinrad is $25 \mathrm{~mm}$, in (b) and (e) the number of pinholes is 10 and pinrad increases from 15 to $35 \mathrm{~mm}$, and in (c) and (f) the number of apertures goes from 9 to 15 and pinrads are 20 and $30 \mathrm{~mm}$ alternatingly.

design with two pinhole circles (see figure 4(c)) to those of the 15 -pinhole design with one pinhole circle, lying in between the previous two, (see figure 4(a)). For central voxels the results are very similar, but the nice reconstruction quality is now also apparant for a larger extent of voxels.

If we put the results for the rat brain phantom (figure 4(d)(f)) next to those of the homogeneous sphere (figure 4(a)-(c)), the same trends are visible and the conclusions drawn above remain valid.

\section{Interpretation of the Effect of Overlap}

In the previous section a lot of multipinhole designs have been evaluated in order to get a feeling of the impact of overlapping projections on the reconstruction image quality. From the results it became clear that it is better to avoid or, if possible, even to remove overlap. However, the amount of overlap was only described qualitatively (high, medium or low). Therefore, a more quantitative approach is proposed here, based on the two overlap quantification measures presented in section II-C.

In order to find out some of the underlying reasons for the preference for non-multiplexing designs, we look more thoroughly at the influence of the variation of the distance between the central and the surrounding pinholes for three different 7-pinhole designs, and at the effect of overlap removal for these designs. In figure 5(a) the CNR is plotted with respect to the examined voxels of the homogeneous sphere, as in the previous section. Figure 5(b) shows the amount of overlap, $\phi_{j}$, in the detector pixels that get information from voxel $j$ (see equation (9)), and figure 5(c) plots the loss of useful sensitivity, $\psi_{j}$, as calculated by equation (10).

From figure 5(b) one can deduct that all voxels are seen with a similar amount of overlap through the design with pinrad $15 \mathrm{~mm}$, whereas only the most excentrical voxels suffer (a little) from overlap for the design with pinrad $35 \mathrm{~mm}$. That is why only for the $15 \mathrm{~mm}$ pinrad design there is a large improvement after overlap removal. After all, removing the multiplexed sensitivity increases the value of the useful sensitivity by eliminating the ambiguity of the information in a detector pixel.

However, the results for the design with pinrad $25 \mathrm{~mm}$ (with overlap), drawn in green, reveal that also a reasonably large amount of overlap occurs at the projection of more excentrical voxels $\left(0.4 \leq \phi_{j} \leq 0.6\right)$. Despite this, overlap removal induces almost no quality improvement. Apparently, the CNR is a very non-linear function of $\phi_{j}$. 


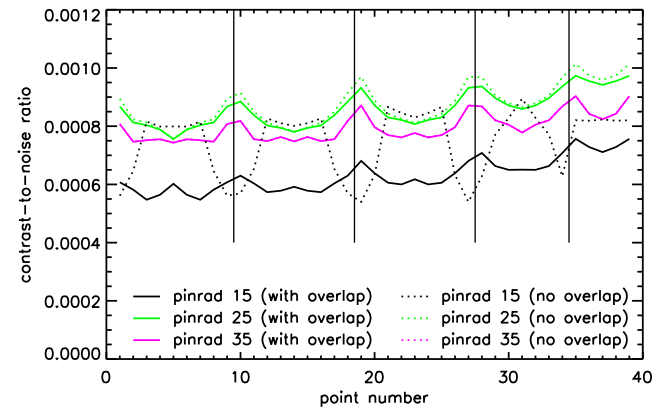

(a) Contrast-to-noise ratio

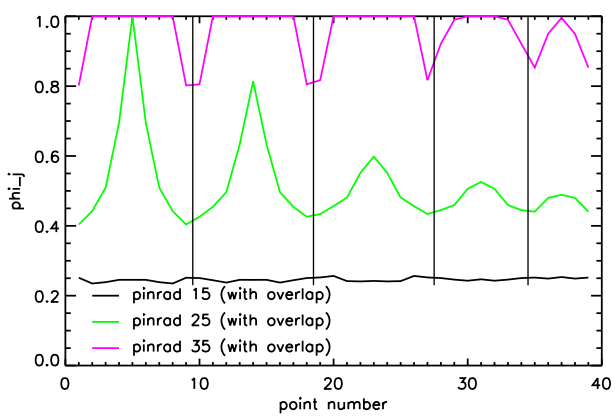

(b) Amount of overlap, $\phi_{j}$

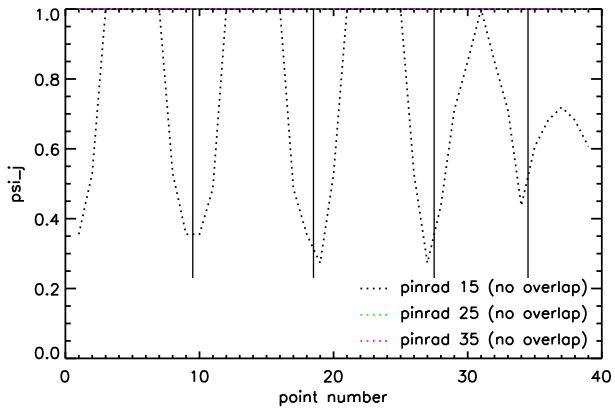

(c) Loss of useful sensitivity after overlap removal, $\psi_{j}$

Fig. 5. Evaluation of three 7-pinhole designs with different degrees of overlap and without overlap to interpret the effect of overlap on the reconstruction image quality of a homogenous sphere. The three graphs plot for each voxel shown in figure 2 their (a) contrast-to-noise ratio, (b) amount of overlap, and loss of useful sensitivity after overlap removal (with the central sampling method).

What is still unclear for the first design at this point, is why the image quality decreases for excentrical voxels, although the degree of multiplexing is more or less equal for all voxels. This can be explained by figure 5(c). During overlap removal useful sensitivity for these excentrical voxels was thrown away. As a consequence, the gain of information is counteracted by the loss of information, which can result in a decrease in image quality, instead of an increase.

\section{Discussion}

The validation study for multipinhole SPECT gave almost identical results for the multiplexing and the non-multiplexing case. It might be more interesting to repeat this study for some designs where the elimination of the overlap does have an important influence. These simulations will be run in the near future.

For single pinhole SPECT designs with different aperture diameters a similar validation study has been performed [7]. Comparable results for the optimal diameter and for the agreement between the approximate and the reference method were found.

From the validation study we could remark that the calculation of the CRC and the variance was more sensitive to the number of interations (for the reference method) and to the choice for $\epsilon$ in equation (7) (for the approximate method) than the CNR. The instability increases with increasing aperture diameter and decreasing number of iterations. In these cases less post-smoothing is allowed, since the resolution of the impulse response is already high with respect to the imposed target resolution. Therefore, the instability problem augments if the shape of the impulse response resembles less an (isotropical) 3D-Gaussian. Fortunately an increase or a decrease in CRC accords with an increase or a decrease, respectively, in the variance, such that it has only a minor impact on the CNR. A possible solution to reduce this instability, is to give more degrees of freedom to the post-smooth filter $P$, e.g. by allowing different FWHM in the three directions or by changing the shape of $P$. However, since the approximation of the CNR is acceptably accurate and in practice we post-smooth with an isotropic Gaussian, we decided to proceed with the CNR as the reconstruction image quality measure and with an isotropic Gaussian as the post-smooth filter.

The calculation of the pseudoinverse $G$ of the Fisher information matrix $F$ includes a factor $\max (\Re(F), 0)$ both in the nominator and in the denominator. In our experience, frequencies ${ }^{5}$ with $\Re(F)<0$ have a negative influence on the accuracy of the approximations. This is probably because they are mostly caused by noise.

Two methods to remove the overlap in the projection images were presented in section II-B. All results shown in this paper were calculated using the second method (central sampling). The first method (maximum sensitivity) gave in most cases similar or sometimes inferior results (in the case of the two pinhole circles with different pinrads). Those results were therefore omitted.

Despite the purely mathematical definition of the overlap removal methods, we believe practical solutions can be found, such as the addition of septa in between the pinhole plate and the detector, since the sensitivity areas corresponding to the

\footnotetext{
${ }^{5}$ In the Fourier domain we shift the image such that the DC-value is positioned at $[0,0,0]$.
} 
different pinhole apertures form non-overlapping closed entities. However, possible effects that might deteriorate the image quality, e.g. badly aligned septa with respect to the pinhole plate or septa penetration, should be taken into consideration.

While searching for a better design, one should keep some things in mind. Firstly, the approximate method to quantify the image quality is space- and object-variant. A well defined problem statement is the first step in the optimization process: for which application will it be used, for which portion of the FOV should the quality be optimized, ... Secondly, it still is an approximate method. Very small improvements could be caused by inaccuracies. Therefore it is useful to verify the quality of (some of) the best designs with the reference method (i.e. using iterative reconstructions). Finally, a trade-off is often necessary between the degree of quality improvement and the feasability of the design.

Another important issue is the positioning of the pinhole apertures in a multipinhole design. A regular pattern as proposed in this work might cause artifacts in the reconstruction image (see [7], [15], [16]) in the form of points (ghost points) or circular shapes (ghost circles). The choice to investigate regular designs in this work was based on the fact that these are a bit easier to simulate. In the future we plan to have a closer look at the effect of these artifacts on the reconstruction quality. It should be verified whether these artifacts are translated adequately into a lower CNR.

In section IV-C a more quantitative approach to evaluate the effect of overlap was proposed. We do not claim, however, that the two presented measures to quantify the overlap have a predictive value. They were only meant to help with the interpretation of the results (CNR) found by the approximate method; not to replace this method.

Our main conclusion that overlap decreases the image reconstruction quality is in agreement with the findings of Meikle et al. [4], Meng et al. [5] and Mok et al. [6].

\section{CONCLUSION}

A method for investigating the effect of overlapping projections on the reconstruction image quality in multipinhole SPECT has been presented. The contrast-to-noise ratio was used as a quality measure and was calculated based on efficient analytical approximations for the properties of the linearized local impulse response. We proposed two intuitive overlap removal methods that assign the sensitivity in each detector pixel to one of the pinholes contributing to the overlap. To evaluate the effect of multiplexing the CNR was calculated for a number of voxels in a homogeneous sphere and in a realistic software rat brain phantom imaged by several multipinhole SPECT designs with different amounts of overlap. The results were very similar for these two phantoms and indicate that the elimination of overlap yields a strong improvement in image quality. Points at the edge of the FOV (especially in the axial direction), however, sometimes benefit from overlap, since overlap removal can reduce the sensitivity for a voxel. In addition, two measures to quantify the overlap were suggested to help with the interpretation of the results.

\section{ACKNOWLEDGMENT}

The authors would like to thank Frank DiFilippo from Cleveland Clinic Foundation (USA) for the interesting discussions at the IEEE NSS-MIC conference in San Diego.

\section{REFERENCES}

[1] S. R. Meikle, R. R. Fulton, S. Eberl, M. Dahlbom, K.-P. Wong, and M. J. Fulham, "An Investigation of Coded Aperture Imaging for Small Animal SPECT.” IEEE Trans. Nucl. Sci., Vol. 48, No. 3, 2001.

[2] F. J. Beekman and B. Vastenhouw, "Design and Simulation of a HighResolution Stationary SPECT system for small animals." Phys. Med. Biol., Vol. 49, pp. 4579-4592, 2004.

[3] N. U. Schramm, G. Ebel, U. Engeland, T. Schurrat, M. Béhé, and T. M. Behr, "High-Resolution SPECT Using Multipinhole Collimation." IEEE Trans. Nucl. Sci., Vol. 50, No. 3, June 2003.

[4] S. R. Meikle, P. Kench, A. G. Weisenberger, R. Wojcik, M. F. Smith, S Majewski, S. Eberl, R. R. Fulton, A. B. Rosenfeld, and M. J. Fulham, "A Prototype Coded Aperture Detector for Small Animal SPECT." IEEE Trans. Nucl. Sci., Vol. 49, No. 5, 2002.

[5] L. J. Meng, W. L. Rogers, N. H. Clinthorne, and J. F. Fessler, "Feasibility Study of Compton Scattering Enhanced Multiple Pinhole Imager for Nuclear Medicine." IEEE Trans. Nucl. Sci., Vol. 50, No. 5, 2003.

[6] S. P. Mok, Y. Wang, and B. M. W. Tsui, "Quantification of the Multiplexing Effect in Multi-Pinhole Small Animal SPECT" IEEE Nucl. Sci. Symp. and Med. Imag. Conf. 2006, M07-1, San Diego, CA, USA, 2006.

[7] K. Vunckx, D. Bequé, M. Defrise, and J. Nuyts, "Single and Multipinhole Collimator Design Evaluation Method for Small Animal SPECT." IEEE Nucl. Sci. Symp. and Med. Imag. Conf. Rec., Vol. 4, pp. 2223 - 2227, 2005.

[8] J. A. Fessler and W. L. Rogers, "Spatial resolution properties of penalizedlikelihood image reconstruction: space-invariant tomographs." IEEE Trans. Image Proc., Vol. 5, No. 9, pp. 1346-1358, 1996.

[9] J. A. Fessler, "Mean and Variance of Implicitly Defined Biased Estimators (such as Penalized Maximum Likelihood): Applications to Tomography." IEEE Trans. Image Proc., Vol. 5, No. 3, pp. 493-506, 1996.

[10] J. Qi and R. M. Leahy, "Resolution and Noise Properties of MAP Reconstruction for Fully 3-D PET." IEEE Trans. Med. Imag., Vol. 19, No. 5, 2000.

[11] J. A. Fessler, "Analytical Approach to Regularization Design for Isotropic Spatial Resolution.", IEEE Nuc. Sci. Symp. and Med. Imag. Conf. Rec., Vol. 3, pp. 2022-2026, 2003.

[12] A. Andreyev, M. Defrise, and C. Vanhove, "Pinhole SPECT Reconstruction Using Blobs and Resolution Recovery." IEEE Trans. Nucl. Sci., Vol. 53, No. 5, pp. 2719-2728, 2006.

[13] M. H. Hudson and R. S. Larkin, "Accelerated image reconstruction using ordered subsets of projection data." IEEE Trans. Med. Imag., Vol. 13, No. 4, pp. 601-609, 1994.

[14] J. A. Fessler, "Spatial resolution and noise tradeoffs in pinhole imaging system design: a density estimationn approach." Optics Express, Vol. 2, No. 5, pp. 237-253, 1998.

[15] G. Bal, G. L. Zeng, R. M. Lewitt, Z. Cao, and P. D. Acton, "Study of different pinhole configurations for small animal tumor imaging." IEEE Nucl. Sci. Symp. and Med. Imag. Conf. Rec., Vol. 5, pp.3133 - 3137, 2004.

[16] Y. Wang and B. M. W. Tsui, "Application of Crosstalk Concept to Assessment of Multi-Pinhole Collimator Designs in Small Animal SPECT Imaging." IEEE Nucl. Sci. Symp. and Med. Imag. Conf. 2006, M16-8, San Diego, CA, USA, 2006. 